\title{
Pesquisa de Listeria monocytogenes e identificação dos sorovares em alimentos prontos para o consumo comercializados no estado do Rio De Janeiro
}

Débora Alves Ferreira da Silva

Mestranda, Laboratório de Alimentos e Saneantes, INCQS/Fiocruz; Av. Brasil, 4365. Manguinhos, Rio de Janeiro-RJ, Brazil.

\section{Carla Trece Carvalho}

Laboratório de Alimentos e Saneantes, INCQS/Fiocruz; Av. Brasil, 4365. Manguinhos, Rio de Janeiro-RJ, Brazil.

\section{Marcelo Luiz Lima Brandão}

Laboratório de Vacinas Virais, Biofármacos e Culturas Celulares, INCQS/Fiocruz; Av. Brasil, 4365. Manguinhos, Rio de Janeiro-RJ, Brazil.

\section{Carla de Oliveira Rosas}

Laboratório de Alimentos e Saneantes, INCQS/Fiocruz; Av. Brasil, 4365. Manguinhos, Rio de Janeiro-RJ, Brazil.

\section{Valéria de Mello Medeiros}

Mestranda, Laboratório de Alimentos e Saneantes, INCQS/Fiocruz; Av. Brasil, 4365. Manguinhos, Rio de Janeiro-RJ, Brazil.

Rodrigo Domingos Overa Tavares Laboratório de Alimentos e Saneantes, INCQS/Fiocruz; Av. Brasil, 4365. Manguinhos, Rio de Janeiro-RJ, Brazil.

\section{Luiza Vasconcellos}

Laboratório de Alimentos e Saneantes, INCQS/Fiocruz; Av. Brasil, 4365. Manguinhos, Rio de Janeiro-RJ, Brazil.

\section{Silvia Maria dos Reis Lopes}

Laboratório de Alimentos e Saneantes, INCQS/Fiocruz; Av. Brasil, 4365. Manguinhos, Rio de Janeiro-RJ, Brazil. 


\section{Resumo}

A listeriose e é considerada uma doença de origem alimentar de grande importância em saúde pública. Devido a diversos fatores que acarretam no aumento da oferta de produtos prontos para o consumo, o objetivo deste estudo foi pesquisar L.monocytogenes nestes alimentos e identificar o sorovar dos isolados. Foram analisadas 67 amostras de alimentos prontos para o consumo comercializados no Rio de Janeiro pela técnica de enriquecimento-seletivo descrito no BAM-FDA e a identificação realizada por PCR. De 67 amostras, 14(20,9\%) apresentaram contaminação por L. monocytogenes, sendo oito(11,9 \%) de queijo tipo minas frescal, quatro(6.0\%) de saladas e duas(3.0\%) de alimentos provenientes da culinária japonesa. Os sorotipos encontrados foram $o$ $1 / 2 \mathrm{a}(\mathrm{n}=1)$, em uma amostra de queijo tipo Minas Frescal, e 1/2b(n=13) nas demais amostras. A presença de L.monocytogenes em alimentos prontos para consumo representa um risco à saúde da população, principalmente os pertencentes aos grupos de risco, como grávidas, idosos e imunossuprimidos.

Palavras-chave: Alimentos prontos para consumo. Listeria monocytogenes. Listeriose. Sorotipagem. Segurança de alimentos. 


\section{Abstract}

Foodborne diseases (FBD) are of major importance in currently world scenario and are classified as a public health problem. Among the major FBD causing microorganisms in the world, Listeria monocytogenes can be present in ready-to-eat foods because of their survival characteristics under adverse environmental conditions. Listeriosis is considered a foodborne disease of great importance in public health due to its seriousness; the high lethality rate (20-30\%) and the propensity of certain individuals to develop the disease. The objective of this study was to investigate, through conventional and molecular techniques, the presence of $L$. monocytogenes in these types of food and to identify the serovar of the isolates. Sixty-seven samples of ready-to-eat foods marketed in the state of Rio de Janeiro were analyzed. RDC 12/2001, which establishes microbiological standards for foods in Brazil, determines the absence of $L$. monocytogenes only in cheese of medium and high humidity. Of the 67 ready-to-eat foods samples analyzed, 14 (20.9\%) samples were contaminated with L. monocytogenes, eight (11.9\%) of cheese Minas type, four $(6.0 \%)$ of salads and two (3.0\%) of foods from japanese cookery. The serotypes found were $1 / 2 a(n=1)$, in a sample of cheese Minas type and $1 / 2 b(n=13)$ in the other samples. The presence of $L$. monocytogenes in ready-to-eat foods represents a risk to the population's health, especially those belonging to the risk groups, such as pregnant women, elderly and immunosuppressed persons. It is necessary to provide guidance to consumers, especially those belonging to risk groups, about the risks involved in the consumption of these foods. A risk analysis of these products should be considered by the Sanitary Surveillance organisms in order to avoid damages to the population`s health due to the consumption of $L$. monocytogenes in contaminated products.

Keywords: Ready-to-eat foods. Listeria monocytogenes. Listeriosis. Serotyping. Food safety. 


\section{Introdução}

Os alimentos prontos para consumo tem ganhado cada vez mais espaço na alimentação dos indivíduos, seja pela praticidade que eles oferecem e/ou pela mudança do estilo de vida das pessoas (NEWELL et al., 2010). A falta de fiscalização e a crescente demanda da indústria por produções cada vez maiores são fatores que interferem na qualidade e segurança dos alimentos (SCHLINDWEIN; KASSOUF, 2007).

As doenças transmitidas por alimentos (DTA) possuem grande importância no cenário mundial atual e são classificadas como um problema de saúde pública, tanto em países desenvolvidos quanto países em desenvolvimento (MARCHI et al., 2011). Dentre os principais microrganismos causadores de DTA no mundo, a Listeria monocytogenes pode estar presente em alimentos prontos para consumo devido a suas características sobrevivência e resistência a condições ambientais adversas (FRANCO; LANDGRAF, 2005; NALÉRIO et al., 2009). Este microrganismo pode se multiplicar sob temperatura de refrigeração e é classificado como um microrganismo psicotrópico, tolerando frequentes congelamentos e descongelamentos, atinge ampla faixa de temperatura que varia entre $1^{\circ} \mathrm{C}$ e $45^{\circ} \mathrm{C}$ e seu crescimento é verificado a $0^{\circ} \mathrm{C}$. É capaz de sobreviver a uma ampla faixa de $\mathrm{pH}(4,1-9,6)$, crescer em ambientes com baixa atividade de água $(<0,93)$ e concentrações de até 10\% de cloreto de sódio (JAY, 2005; JEYALETCHUMI et al., 2010).

A L. monocytogenes é uma bactéria oportunista, considerando que a infecção provém, dentre outros fatores, das condições imunológicas dos indivíduos (CRUZ, et al., 2008). Este patógeno tem a capacidade de sobreviver e se multiplicar em meios com poucos nutrientes, intracelularmente e fora do organismo dos hospedeiros, animais e homem (GERMANO e GERMANO, 2013). A virulência das cepas também parece estar relacionada ao sorovar, uma vez que determinados grupos de sorotipos estão mais associados a casos de infecções em humanos (KATHARIOU, 2002; JAY, 2005).

L. monocytogenes possui 13 grupos sorológicos $(1 / 2 \mathrm{a}, 1 / 2 \mathrm{~b}, 1 / 2 \mathrm{c}, 3 \mathrm{a}, 3 \mathrm{~b}, 3 \mathrm{c}, 4 \mathrm{a}$, $4 \mathrm{ab}, 4 \mathrm{~b}, 4 \mathrm{c}, 4 \mathrm{~d}, 4 \mathrm{e}, 7)$, sendo os sorotipos $1 / 2 \mathrm{a}, 1 / 2 \mathrm{~b}$ e $4 \mathrm{~b}$ os mais relevantes epidemiologicamente, representando $95 \%$ dos surtos desencadeados pela bactéria em humanos (CAMARGO, 2013; FORSYTHE, 2013). A listeriose é considerada uma doença de origem alimentar de fundamental importância em saúde pública, devido a sua 
gravidade; a elevada taxa de letalidade (20-30\%) e a propensão de determinados indivíduos desenvolverem a doença. $\mathrm{O}$ extenso período de incubação do microrganismo prejudica a identificação do agente causador e a rastreabilidade da origem da contaminação do alimento (CHIARINI, 2007; BARANCELLI, 2010).

Os indivíduos mais propensos a listeriose são as gestantes, os fetos, os neonatos, os idosos e os indivíduos imunocomprometidos, como os portadores do vírus da imunodeficiência humana (HIV), de câncer, indivíduos transplantados, diabéticos, cirróticos, asmáticos, com colite ulcerativa e aqueles que realizam tratamento com medicamentos, como quimioterápicos e corticosteroides (COSTA, 2010; FORSYTHE, 2013). Aproximadamente um terço das infecções acomete as gestantes (BARANCELLI, 2010), que apresentam risco 14 vezes maior de adquirir a doença após o consumo de alimentos contaminados quando comparadas a indivíduos saudáveis (COSTA, 2010). Em indivíduos adultos com idade inferior a 60 anos, a letalidade está em 25\%, e naqueles com idade superior, 40\%. Aproximadamente $84 \%$ dos casos ocorrem antes de 50 anos de idade, e 40\% após os 70 anos (GERMANO e GERMANO, 2013). Em casos de meningite ocasionada por L. monocytogenes o índice de mortalidade pode atingir $70 \%$ e de $50 \%$ para septicemias. Em infecções perinatais, esse índice aumenta ultrapassando 80\% (FORSYTHE, 2013).

A ocorrência de listeriose no Brasil é considerada subdiagnosticada e subnotificada (DESTRO, 2006), não havendo relatos da doença procedente do consumo de alimentos contaminados reconhecidos no país. Entretanto, pesquisas realizadas comprovam a presença de $L$. monocytogenes em inúmeros produtos alimentícios (CRUZ et al., 2008; BARANCELLI, 2010; BRANDAO et al., 2013; MORENO, 2013; SILVA, 2016).

Considerando a complexidade das DTA no contexto da saúde pública e da economia mundial, é de suma importância o conhecimento dos agentes etiológicos envolvidos em surtos e seus efeitos relacionados. Devido a diversos fatores que acarretam em um grande consumo de produtos prontos para o consumo, o objetivo deste estudo foi pesquisar L. monocytogenes nestes tipos de alimentos. 


\section{Material e Métodos}

\section{AMOSTRAGEM}

Um total de 67 amostras de alimentos prontos para consumo foi analisado, incluindo: 15 amostras de produtos cárneos (embutidos ou não), 15 amostras de queijos tipo Minas frescal, 15 amostras de saladas mistas, e 22 amostras de alimentos provenientes da culinária japonesa (sushis, sashimis, entre outros), comercializadas em restaurantes e estabelecimentos dos municípios do Rio de Janeiro e Niterói do estado do Rio de Janeiro, no período de abril a junho de 2016. As amostras foram acondicionadas em embalagens estéreis individuais para transporte e mantidas sob temperatura de refrigeração $\left(4-8^{\circ} \mathrm{C}\right)$ até o momento da análise.

\section{ANÁLISES MICROBIOLÓGICAS}

A pesquisa de L. monocytogenes foi realizada conforme descrito por Hitchins (2003). Vinte e cinco gramas da amostra foram homogeneizadas em $225 \mathrm{~mL}$ caldo de enriquecimento tamponado para Listeria (Oxoid, Basingstoke, Inglaterra). O homogenato foi incubado a $30^{\circ} \mathrm{C}$ por $4 \mathrm{~h}$. Após este período foram adicionados os agentes seletivos (acriflavina, ácido nalidíxico e ciclohexamina). A incubação a $30{ }^{\circ} \mathrm{C}$ foi mantida por um total de $48 \mathrm{~h}$. Para o isolamento foi utilizado o Chromogenic Listeria Agar (Oxoid, Basingstoke, Inglaterra). As colônias características foram submetidas aos seguintes testes morfológicos e fenotípicos: coloração de Gram, fermentação de carboidratos (glicose, esculina, maltose, ramnose, manitol e xilose), produção de catalase, hemólise em ágar sangue de carneiro e motilidade.

\section{CONFIRMAÇÃO POR REAÇÃO EM CADEIA DE POLIMERASE (PCR)}

As amostras que apresentaram perfil característico de L. monocytogenes nos testes morfológicos e fenotípicos foram submetidas à técnica de reação em cadeia pela polimerase (PCR) com alvo no gene que codifica a internalina $\mathrm{AB}$, segundo o protocolo descrito por JUNG (2001). Foi realizada a extração de DNA das culturas com uso do kit 
Dneasy Blood \& Tissue (Qiagen, EUA), de acordo com as instruções do fabricante. A PCR foi preparada em um volume total de $25 \mu \mathrm{L}$ contendo: $2,5 \mu \mathrm{L}$ de DNA molde (20-60 ng/ $\mu 1), 25$ pmol dos iniciadores AB1- 5'-CTTCAGGCGGATAGATTAGG3' e AB3 5'-TTCGCAAGTGAGCTTACGTC-3') e PCR MasterMix 1X (ThermoScientific, EUA). A amplificação foi realizada em aparelho SimpliAmp ThermalCycler (Applied Biosystems, Singapore) nas seguintes condições: 94º C/3 min; 25 ciclos de $94^{\circ} \mathrm{C} / 30 \mathrm{~s}, 62^{\circ} \mathrm{C} / 30 \mathrm{~s} \mathrm{e} 72^{\circ} \mathrm{C} / 1 \mathrm{~min}$; e extensão final a $72^{\circ} \mathrm{C} / 5 \mathrm{~min}$. Os produtos amplificados foram submetidos à eletroforese em gel de agarose a 1,5\% a 100 V/50 min. Após, o gel foi corado em solução de brometo de etídio 0,5 $\mu \mathrm{g} / \mathrm{mL}$ (Sigma, EUA) por 15 min e visualizado em analisador de imagens (GE-Healthcare, Inglaterra).

\section{SOROTIPAGEM DOS ISOLADOS DE L. MONOCYTOGENES}

As culturas foram cultivadas em Ágar Tripticase de Soja (TSA) contendo extrato de levedura (Merck, Darmstadt, Germany) a $35^{\circ} \mathrm{C}$ por $48 \mathrm{~h}$ e enviadas ao Laboratório de Zoonoses Bacterianas do Instituto Oswaldo Cruz da Fundação Oswaldo Cruz (IOC/Fiocruz) para identificação e sorotipagem convencional por aglutinação segundo SEELIGER e HÖHNE (1979).

\section{Resultados e Discussão}

De 67 amostras de alimentos prontos para consumo analisadas, 14 (20,9\%) apresentaram contaminação por L. monocytogenes, sendo oito $(11,9 \%)$ de queijo tipo minas frescal, quatro $(6,0 \%)$ de saladas e duas $(3,0 \%)$ de alimentos provenientes da culinária japonesa (Tabela 1). Todos os isolados que apresentaram resultados característicos nos testes morfológicos e fenotípicos foram confirmados como $L$. monocytogenes pela reação de PCR. Após a confirmação, os isolados foram identificados como pertencentes aos sorotipos1/2a $(n=1)$ e $1 / 2 b(n=13)$ (Tabela 1$)$. 
Tabela 1 - Resultados obtidos na pesquisa de L. monocytogenes

\begin{tabular}{l|l|l}
\multicolumn{1}{c|}{$\begin{array}{c}\text { Categoria de produto } \\
\left(\mathbf{n}^{\mathbf{o}} \text { de amostras) }\right.\end{array}$} & \multicolumn{1}{|c}{$\begin{array}{c}\text { No de amostras positivas } \\
(\%)\end{array}$} & \multicolumn{1}{c}{$\begin{array}{c}\text { Identificação dos sorotipos } \\
\left(\mathbf{n} .^{\circ} \text { cepas }\right)\end{array}$} \\
\hline Produtos cárneos (15) & $0(0)$ & $\mathrm{NR}^{\mathrm{a}}$ \\
Blanquet de peru (2) & $0(0)$ & \\
Blanquet de peru light (3) & $0(0)$ & \\
Mortadela (4) & $0(0)$ & \\
Peito de frango (1) & $0(0)$ & \\
Peito de peru (2) & $0(0)$ & $1 / 2 \mathrm{~b}(4)$ \\
Presunto (2) & $0(0)$ & $1 / 2 \mathrm{a}(1) 1 / 2 \mathrm{~b}(7)$ \\
Presunto cozido (1) & $0(0)$ & $1 / 2 \mathrm{~b}(2)$ \\
\hline Salada mista (15) & $4(26,7)$ & \\
\hline Queijo tipo Minas Frescal (15) & $8(53,5)$ & $1 / 2 \mathrm{a}(\mathrm{n}=1), 1 / 2 \mathrm{~b}(\mathrm{n}=13)$ \\
\hline Alimentos provenientes da culinária & $2(9,1)$ & \\
japonesa (22) & & \\
\hline Total (67) & $14(20,9)$ & \\
\hline
\end{tabular}

$\mathrm{NR}^{\mathrm{a}-\mathrm{N} a ̃ o ~ r e a l i z a d o ~}$

A maior ocorrência de L. monocytogenes foi identificada em amostras de queijos tipo Minas Frescal, onde 53,5\% das amostras analisadas estavam contaminadas. A legislação brasileira prevê ausência em $25 \mathrm{~g}$ de amostras de queijos de alta umidade (BRASIL, 2001), desta forma, estas oito amostras foram consideradas em condições sanitárias insatisfatórias segundo os critérios da legislação vigente. Este resultado demonstra que, apesar do parâmetro ser contemplado na legislação (BRASIL, 2001), falhas no controle da qualidade para a liberação de lotes de queijos tipo Minas Frescal podem ocorrer, o que representaria um risco para a população consumidora. Outros trabalhos também verificaram a presença do microrganismo neste tipo de alimento. Silva (2016) constatou a presença de L. monocytogenes em 13,3\% das 30 amostras de queijos tipo Minas Frescal analisadas. Em um estudo ocorrido na região da Zona da Mata mineira, Apolinário e colaboradores (2014), observaram a presença de $L$. monocytogenes em 9,6\% das 31 amostras de queijo tipo Minas Frescal analisadas.

$\mathrm{Na}$ análise de alimentos provenientes da culinária japonesa, 9,1\% das amostras apresentaram contaminação (Tabela 1). A presença de L. monocytogenes em produtos similares já havia sido relatada por Montanari e colaboradores (2015) em um estudo 
envolvendo três estabelecimentos no município de Ji-Paraná, no estado de Rondônia. O grupo detectou a presença do microrganismo em dois estabelecimentos. $\mathrm{O}$ trabalho analisou 15 amostras de sashimi de salmão in natura, sendo cinco amostras de cada estabelecimento. L. monocytogenes foi identificada em uma amostra no estabelecimento 1 e em três amostras no estabelecimento 3. Outros trabalhos envolvendo análise de pescados que podem ser consumidos sem cocção também constataram a presença de $L$. monocytogenes em elevados níveis de contaminação (MANTILLA et al., 2007; PINTO et al., 2010) comprovando o risco potencial que estes alimentos possuem de serem transmissores de doenças alimentares.

$\mathrm{Na}$ análise dos produtos cárneos fatiados não foi detectada a presença de $L$. monocytogenes em nenhuma amostra. Este resultado foi similar ao encontrado por Sousa e colaboradores (2016), que num estudo envolvendo 35 amostras de produtos cárneos fatiados detectaram apenas uma $(2,8 \%)$ amostra contaminada por L. monocytogenes. Esses resultados sugerem que as técnicas empregadas na indústria, como processamento térmico destes alimentos, são efetivas para eliminação do patógeno (VITAS; GARCIAJALON, 2004).

A análise de saladas apresentou um índice de $26,7 \%$ das amostras contaminadas por L. monocyogenes. Brandão e colaboradores (2013) detectaram a presença do microrganismo em 16,7\% de 30 amostras de alface de serviços de alimentação. A presença de L. monocytogenes em saladas prontas para consumo já foi relatada em várias partes do mundo e é evidente a necessidade de cuidados na manipulação e higienização destes alimentos para redução dos riscos de contaminação (AJAYEOBA, et al., 2016; CHAU et al., 2017; CHEN et al., 2017).

O sorotipo mais encontrado foi o $1 / 2 \mathrm{~b}(92,8 \%)$, que está entre os três principais sorotipos patogênicos de L. monocytogenes. Este sorotipo é amplamente encontrado em alimentos e ambientes industriais. Nalério e colaboradores (2009) observou que 51,6\% (16/31) dos isolados de L. monocytogenes de um abatedouro de aves pertenciam ao sorotipo 1/2b, no Rio Grande do Sul. Kovacevic e colaboradores (2013) também encontraram em amostras de produtos prontos para o consumo a base de peixe, no varejo de Vancouver, British Columbia, 5\% das amostras apresentaram contaminação pelos os sorotipos $1 / 2 a(n=3)$ e $1 / 2 b(n=3)$.

Martins (2008) analisou 130 amostras de dois grupos de produtos cárneos (presunto e salame) e o sorotipo $1 / 2 \mathrm{~b}$ foi o segundo mais encontrado com uma 
frequência de $25 \%$ nestes produtos. O sorotipo $1 / 2 \mathrm{~b}$ já foi responsável por surtos pelo mundo, como o que ocorreu entre janeiro de 2013 e fevereiro de 2014 no norte da Espanha (PÉREZ-TRALLERO et al., 2014). Foram relatados 27 episódios, incluindo 11 casos relacionados à gravidez sendo o microrganismo envolvido uma cepa de $L$. monocytogenes sorotipo $1 / 2 \mathrm{~b}$.

Os sorotipos $1 / 2 \mathrm{a}, 1 / 2 \mathrm{~b}$ e $1 / 2 \mathrm{c}$ são comumente isolados em alimentos (SWAMINATHAN; GERNER-SMIDT, 2007). Brandão e colaboradores (2013) ao analisarem hortaliças in natura e prontas para consumo comercializadas no município do Rio de Janeiro, obtiveram oito isolados pertencentes ao sorotipo 1/2a. Brito e colaboradores (2008) também detectaram cepas de L. monocytogenes sorotipo 1/2a ( $\mathrm{n}=6)$ em amostras de queijo tipo Minas Frescal.

\section{Considerações finais}

A RDC n. 12 (BRASIL, 2001) que estabelece padrões microbiológicos para alimentos, determina a ausência de $L$. monocytogenes apenas em queijos de média e alta umidade. Porém, não constitui parâmetros para este patógeno em sushi, sashimi e nem em saladas prontas. Já em países desenvolvidos como Estados Unidos e Europa o critério para L. monocytogenes é ausência em todos os produtos prontos para consumo (UNIÃO EUROPÉIA, 2005; ORSI; DEN BAKKER; WIEDMANN, 2011). Pelo fato destes alimentos, muitas vezes, serem consumidos com total ausência de tratamento térmico e, em alguns casos por pessoas com algum tipo de deficiência imunológica, a presença do patógeno detectada nestes tipos de alimentos neste estudo pode representar um sério risco a estes indivíduos. É necessária a orientação dos consumidores, principalmente aos pertencentes aos grupos de risco, sobre os perigos existentes no consumo destes alimentos. 


\section{Referências}

AJAYEOBA, T. et al.The incidence and distribution of Listeria monocytogenes in readyto- eat vegetables in South- Western Nigeria. Food Sciene E' Nutrition, v. 4, n. 1, p. 5966, 2016.

APOLINÁRIO, T. C. C.; SANTOS, G. S.; LAVORATO, J.A.A. Avaliação da qualidade microbiológica do Queijo minas frescal produzido por Laticínios do estado de minas gerais. Revista do Instituto de Laticinios Cândido Tostes, v. 69, n. 6, p. 433-442, 2014.

BARANCELLI, G, V. Ocorrência e caracterização sorológica e genotípica de Listeria monocytogenes em indústrias de queijo do Estado de São Paulo. 2010. 115 f. Tese (Doutorado em Zootecnia) - Faculdade de Zootecnia e Engenharia de Alimentos, Universidade de São Paulo, Pirassununga, 2010.

BRANDÃO, M. L. L. et al. Listeria monocytogenes em hortaliças: isolamento e sorotipagem. Revista Instituto Adolfo Lutz, v. 72, n. 1, p. 116-121, 2013.

BRASIL. Ministério da Saúde. Agência Nacional de Vigilância Sanitária. Resolução RDC n. ${ }^{\circ} 12$, de 02 de Janeiro de 2001. Aprova o regulamento sobre padrões microbiológicos para alimentos e seus Anexos I e II. Diário Oficial [da] República Federativa do Brasil, Brasília, Distrito Federal, n. 7, 10 Jan. 2001. Seção 1, p. 45-53.

CAMARGO, A.C. Caracterização fenotipica e molecular de isolados de Listeria moncytogenes obtidos em uma planta de carne bovina. 2013. 73 f. Dissertação (Mestrado em Medicina Veterinária) - Universidade Federal de Viçosa, Viçosa, 2013.

CHAU, M. L. et al. Microbial survey of ready-to-eat salad ingredients sold at retail reveals the occurrence and the persistence of Listeria monocytogenes Sequence Types 2 and 87 in pre-packed smoked salmon. BMC Microbiology, v.17, n 46, 2017.

CHEN, Y. et al. Singleton ST382, an emerging clonal group of Listeria monocytogenes associated with three multistate outbreaks linked to contaminated stone fruit, caramel apples and leafy green salad. Journal of Clinical Microbiology, v.55, n.3, p. 931-941, 2017.

CHIARINI, E.B. Listeria monocytogenes em matadouros de aves: marcadores sorológicos e genéticos no monitoramento de sua disseminação. 2007. 145 f. Tese (Doutorado em Ciência 
dos Alimentos) - Faculdade de Ciências Farmacêuticas, Universidade de São Paulo, São Paulo, 2007.

COSTA, C. A. R. Avaliação da expressão do consumidor à Listeria monocytogenes, Salmonella spp. Campylobacter spp.e Escherichia coli produtora de toxina de Shiga em produtos cárneos refrigerados comercializados no município de São Paulo. 2010. 112 f. Tese (Doutorado em Ciência dos Alimentos) - Faculdade de Ciências Farmacêuticas, Universidade de São Paulo, São Paulo, 2010.

CRUZ, C. D.; MARTINEZ, M. B.; DESTRO, M. T. Listeria monocytogenes: um agente infeccioso ainda pouco conhecido no Brasil. Alimentos e Nutrição, Araraquara, v. 19, n. 2, p.195-206, Abr./Jun. 2008.

DESTRO, M. T. Listeria monocytogenes na cadeia produtiva de alimentos: da produção primária ao consumidor final. 2006. 74f. Tese (Livre Docência) - Faculdade de Ciências Farmacêuticas, Universidade de São Paulo, São Paulo, 2006.

FORSYTHE, S. J. Microbiologia da segurança dos alimentos. Porto Alegre: Artmed, 2a ed., 607p., 2013.

FRANCO, B. D.G.M.; LANDGRAF, M. Microbiologia dos Alimentos. São Paulo: Atheneu, 2005. 182 p.

GERMANO, P. M. L.; GERMANO, M. I .S. Sistema de Gestão: qualidade e segurança dos alimentos. São Paulo: Manole, 2013.

HITCHINS, A.D. Detection and Enumeration of Listeria monocytogenes in Food. In: Bacteriological analytical manual Online. [S.1]; FDA, 2003. Disponível em http://www.cfsan.fda.gov/ ebam/bam-10.html. Acesso em: 06 jun. 2017.

JAY, J. M. Microbiologia de alimentos. Porto Alegre: Artmed, 2005.

JEYALETCHUMI, P. et al. Review article: Detection of Listeria monocytogenes in foods. International Food Research Journal, v.17, p. 1-11, 2010.

JUNG, Y. Detection of Listeria monocytogenes using genetic and Immunological tools. Athens, Georgia, 2001. 
KATHARIOU, S. Listeria monoytogenes virulencia e pathogenicity, a Food Safety Perspective. Journal Food Protection. v. 65, n. 11, p. 1811-1829, 2002.

KIVACEVIC, J.; MESAK, L.R.; ALLEN, K.J. Ocurence and characterization os Listeria spp. In ready-to-eat retail foods from Vancouver, British Columbia. Food Microbiology, v. 30, p- 372-378, 2012.

MANTILLA, S. P. S. et al. Importância da Listeria monocytogenes em alimentos de origem animal. Revista da FZVA, v.14, p. 180-192. 2007.

MARCHI, D. M. ; BAGGIO, N.; TEO, C. R. P. A. Ocorrência de surtos de doenças transmitidas por alimentos no Município de Chapecó, Estado de Santa Catarina, Brasil, no período de 1995 a 2007. Epidemiologia e Serviços de Saúde, Brasília, v. 20, n. 3, p.401407, jul./set. 2011.

MARTINS, E. A. Listeria monorytogenes em produtos fatiados do tipo ready-to-eat, presunto cozido e salame, comercializados no Município de São Paulo, ocorrência, quantificação e sorotipagem, 2008. Tese (doutorado), faculdade de Saúde Pública da USP, São Paulo.

MONTANARI, A. S. et al. Avaliação da qualidade microbiológica de sashimis de salmão, preparados e comercializados em restaurantes japonês no município de JiParaná - RO. South American Journal of Basic Education, Technical and Technological. v. 2, n. 1, p. 4-16, 2015.

MORENO, L. Z. Pesquisa de genes de virulência em cepas de Listeria monocytogenes e Listeria innocua originárias de carne suina e ambiente de abatedouros e açougue. 2013. $107 \mathrm{f}$. Dissertação (Mestrado em Saúde Pública) - Faculdade de Saúde Pública, Universidade de São Paulo, São Paulo, 2013.

NALÉRIO, E. S. et al. Listeria monocytogenes: monitoramento desse perigo biológico na cadeia produtiva de frangos do sul do Rio Grande do Sul. Ciência e Tecnologia de Alimentos, Campinas, v. 29, n. 3, p.626-630, Jul./set. 2009.

NEWELL, D.G. et al. Food-borne diseases - The challenges of 20 years ago still persist while new ones continue to emerge. International Journal Food Microbiology. v. 139, (Supl. 1)p. S3-S15, 2010. 
ORSI, R.H.; DEN BAKKER, H.C.; WIEDMANN, M. Listeria monocytogenes lineages: Genomics, evolution, ecology, and phenotypic Characteristics. Mini review. International Journal of Medical Microbiology. v.31, p.79-96, 2011.

PÉREZ-TRALLERO, E. et al. Two Outbreaks of Listeria monocytogenes Infection, Northern Spain. Emerging Infectious Diseases journal, v. 20, n. 12, p. 2155-2157, 2014.

PINTO, A. et al. Occurrence of Listeria monocytogenes in ready-to-eat foods from supermarkets in Southern Italy. New Microbiologica, v. 33, p. 249-252, 2010.

SCHLINDWEIN, M. M.; KASSOUF, A. L. Mudanças no padrão de consumo de alimentos tempo-intensivos e de alimentos poupadores de tempo, por região do Brasil. In: IPEA. (ORG.). Gasto e consumo das famílias brasileiras contemporâneas, 2007, v. 2.

SEELIGER, H. P. H.; HÖHNE, K. Serotyping of Listeria monocytogenes and related species. Methods Microbiology, [S.1.], v. 13, p. 31-49, 1979.

SILVA, D.A.F. Análise microbiológica amostras de queijo minas frescal comercializadas no estado do Rio de Janeiro. 2016. 39 f. Trabalho de conclusão do curso (Especialista em Vigilância Sanitária) - Programa de Pós-Graduação em Vigilância Sanitária, Instituto Nacional em Controle de Qualidade em Saúde, Fundação Oswaldo Cruz, Rio de Janeiro, 2016.

SOUSA, I. S. et al. Monitorização microbiológica em produtos de charcutaria cozidos, fatiados em talhos. Instituto Nacional de Saúde Doutor Ricardo Jorge, Portugal, n. 12 , 2016.

SWAMINATHAN, B.; GERNER-SMIDT, P. The epidemiology of human listeriosis. Microbes and Infection, v. 9, n. 10, p. 1236-1243, 2007.

UNIÃO EUROPÉIA. Commission Regulation (EC) No 2073/2005, 15 de novembro de 2005. Official Journal of the European Union. Bruxelas, 15 nov. 2005.

VITAS, A. L.; GARCIA-JALON, V.A. Ocurrence of Listeria monocytogenes in fresh and processed foods in Navarra (Spain). Internacional Journal Food Microbology, v. 90, n. 3, p. 349-356, 2004. 